Paediatr. Paedolog. 2020 · 55 (Suppl 2):S58-S61 https://doi.org/10.1007/s00608-020-00779-0

(c) Der/die Autor(en) 2020

\section{Matthias Beck}

Katholisch-theologische Fakultät, Institut für Systematische Theologie und Ethik, Theologische Ethik, Forschungsschwerpunkt Medizinethik, Universität Wien, Wien, Österreich

\title{
Ethische Fragen zum Kindeswohl: Gelingen und Misslingen von Entwicklung
}

\section{Kindeswohl}

Der Begriff des Kindeswohls ist schwer definierbar. Einige Zeit hat die österreichische Bioethikkommission, deren Mitglied ich bin, über das Kindeswohl diskutiert, und zwar anlässlich der immer weiter fortschreitenden Möglichkeiten der In-vitro-Fertilisation mit der Möglichkeit der Eizellspende, der Kinder für lesbische Paare und der Präimplantationsdiagnostik. In diesem Beitrag soll es mehr um das Kindeswohl im Hinblick auf die Erziehung, Bildung und die Bedürfnisse des heranwachsenden Kindes gehen. Was die Erziehung angeht, so sind unter anderem die Bücher von Astrid Lindgren zu erwähnen.

Ihre Bücher wurden 1949 zum ersten Mal ins Deutsche übersetzt. In ihnen ging es unter anderem darum, festzuhalten, dass Kinder damals eher klein gehalten und auch mit Schlägen und Gewalt „dressiert“ wurden. Lindgren sagt - festgemacht an Pippi Langtrumpf und dem „entfesselten Kind“ -, dass das Kind sich frei entwickeln können müsse. In dieser Zeit begann das Ende der Gewalt in der Kindererziehung. Das heißt nicht, dass Kinder alles dürfen, wenn keine Gewalt mehr angewendet wird. Aber man hat angefangen, sich Gedanken zu machen über das körperliche, seelische und geistige Wohl der Kinder.

Das Wohl der Kinder hat auch mit dem Begriff der Menschenwürde zu tun. Die Menschenwürde gilt für alle Menschen. Aber sie ist insbesondere für Kinder wichtig, da diese besonderes vulnerabel sind. Der Menschenwürdebegriff wurde 1949 festgeschrieben im Artikel 1 des Deutschen Grundgesetzes und später in der Grundrechtecharta des Europäischen Vertrags von Lissabon. Aus ihm folgt das Recht auf Leben und körperliche sowie geistige Unversehrtheit. Auch in die Kinderrechtskonvention der Vereinten Nationen wurde 1989 hineingeschrieben, dass das Kindeswohl Vorrang haben muss gegenüber anderen Interessen. Es gibt ein Diskriminierungsverbot von Kindern aufgrund von Rasse, Herkunft, Religion, genetischer Ausstattung sowie das Recht auf Leben, Entwicklung und Gesundheit. Ferner gibt es ein Partizipationsrecht, das darauf abzielt, Kinder ernst zu nehmen und sie je nach Alter und Einsichtsfähigkeit in Entscheidungen einzubeziehen und ihre Stimme zu hören. Ferner gibt es ein Recht auf Namen und Staatszugehörigkeit, Recht auf Bildung und Ausbildung, Freizeit, Spiel und Erholung, das Recht sich zu informieren, sich mitzuteilen, gehört zu werden und sich zu versammeln. Weiterhin gibt es ein Recht auf Privatsphäre und gewaltfreie Erziehung im Sinn der Gleichberechtigung. Schließlich gibt es ein Recht auf Familie, elterliche Fürsorge und ein sicheres Zuhause inklusive ein Recht auf Betreuung bei Behinderung. ${ }^{1}$

Das Kindeswohl ist immer wieder gefährdet durch schlechte soziale Umfelder, durch seelische und körperliche Gewalt, aber auch durch fehlende Zeit im Umgang mit Kindern. Es gibt eine Logik der Liebe, die Zeit aufbringt für ein ko-

\footnotetext{
1 Übereinkommen über die Rechte des Kindes. UN-Kinderrechtskonvention, am 26.01.1990 von der Bundesrepublik Deutschlandland unterzeichnet.
}

operatives Zusammensein mit Kindern (Spielen, Sport, Lernen, Diskutieren). Auch strukturelle Rücksichtslosigkeiten gegenüber der Familie gefährden das Wohl von Kindern. Kinder sind nicht nur besonders verletzlich, sondern übernehmen auch später Verantwortung für das Gemeinwohl. So geht es bei der Erziehung von Kindern und Jugendlichen auch um das zukünftige Wohl einer Gesellschaft.

\section{Grundbedürfnisse von Kindern}

Brazelton und Greenspan [1] haben sieben Grundbedürfnisse von Kindern zusammengetragen: Erstens geht es um „das Bedürfnis nach beständigen liebevollen Beziehungen" [1, S. 31-108]. Stabile Beziehungen sind zentral für die gute Entwicklung von Kindern. Sie sind wichtig für die Fremdwahrnehmung, Selbstwahrnehmung, Urteilsbildung und Gewissensbildung. Wichtig dabei ist, dass Eltern sich um ihre Kinder kümmern, mit ihnen Zeit verbringen, spielen, Feste feiern, diskutieren. Denn sonst besteht die Gefahr, dass Kinder viel allein sind und oft im Internet surfen. Wenn Kinder aus der Schule kommen und sofort im Internet spielen, ist im Gehirn oft alles gelöscht, was sie morgens im Unterricht gelernt haben. Das Gehirn braucht Ruhe und Zeit, damit das Gelernte sich setzen und im Gehirn engrammiert werden kann. Auch die Wiederholung des Gelernten ist von großer Bedeutung: repetitio est mater studiorum, die Wiederholung ist die Mutter allen Lernens.

Volksschullehrer sagen, dass Kinder durch das „Leben“ in virtuellen Welten 
auch zunehmend empathieunfähig werden. Sie können kaum noch Freundschaften in der realen Welt schließen. Durch dieses Internetspielen sind keine konkreten Personen mehr da, die sie anfassen und denen sie in die Augen schauen können. So kann auch die Aggression zunehmen, wenn anonym Botschaften in den sozialen Netzwerken verschickt werden können. Womöglich fördert der viele Internetkonsum auch die Demenz (vgl. da$\mathrm{zu}[2])$.

Zweitens geht es um „das Bedürfnis nach körperlicher Unversehrtheit, Sicherheit und Regulation“" [1, S. 109-146]. Aus der Philosophie von Immanuel Kant, die unter anderem darüber nachdenkt, dass jedem Menschen Menschenwürde zukommt, folgt das Recht auf Leben und körperliche Unversehrtheit - wie erwähnt ist dies im Deutschen Grundgesetz und der Grundrechtecharta des Lissaboner Vertrags für Europa festgeschrieben. Dies ist auch für die Medizin ist von großer Bedeutung, denn jeder invasive Eingriff, wie z.B. eine Blutabnahme, ist eine Versehrung des Körpers und bedarf der ausdrücklichen Zustimmung des Patienten. Man nennt dies den „informed consent“. Dieser ist für die Kinderheilkunde von besonderem Wert, da hier die Eltern für die Kinder im Sinn ihrer Fürsorgepflicht entscheiden müssen.

Drittens geht es um „das Bedürfnis nach Erfahrungen, die auf individuelle Unterschiede zugeschnitten sind" [1, S. 147-201]. Gerade die neuzeitliche europäische Philosophie ist sehr auf das Individuum ausgerichtet. Der Subjektcharakter des Menschen tritt mit Beginn der Neuzeit immer mehr hervor. Konkret geht es darum, den Einzelnen als den Einzelnen in seiner individuellen Besonderheit wahrzunehmen und ihm gerecht zu werden. Gerade Kinder spüren sehr, ob sie in ihrer individuellen Entwicklung ernst genommen werden. $\mathrm{Zu}$ viel Behütung und Verwöhnung kann allerdings schaden, weil womöglich keine individuellen Erfahrungen mehr gemacht werden können. Von den Kindern sollten ihre je einmaligen Erfahrungen in den Diskurs eingebracht werden können.

Viertens geht es um „das Bedürfnis nach entwicklungsgerechten Erfahrun- gen“" [1, S. 203-245]. Die Anforderungen an Kinder sollen altersentsprechend sein. Man sollte Kinder nicht in Rollen drängen, die ihrer Altersentwicklung nicht entsprechen, sie also z. B. nicht zu früh mit Erziehungsaufgaben für kleine $\mathrm{Ge}$ schwister betrauen.

Fünftens gibt es „das Bedürfnis nach Grenzen und Strukturen“" [1, S. 247-268]. Kinder haben nicht das Bedürfnis, ohne Begrenzungen zu leben. Sie brauchen Grenzen, die dialogisch sinnvoll mit Fürsorge und Liebe vermittelt werden sollten, nicht einfach mit Strafe. Es geht um die Fähigkeit von Eltern, mit einem Kind kindgerecht argumentieren zu können. Nicht hilfreich ist eine falsche Autorität, die von außen alles durchsetzen will. Wahre Autorität (von „augere“: wachsen lassen, mehren, fördern) bemüht sich darum, in den Kindern das zur Entfaltung zu bringen, was in ihnen angelegt ist (vgl. dazu [3]). Es geht darum, Kindern zu ermöglichen, in ihr Eigenes hineinwachsen zu können.

Sechstens haben Kinder „das Bedürfnis nach stabilen unterstützenden Gemeinschaften und nach kultureller Kontinuität“ [1, S. 269-293]. Es geht darum, Kindern über die Familien hinaus Freundschaften, gute soziale Kontakte und soziale Integration zu ermöglichen. Dies wird sie stabiler machen im späteren Berufsleben.

Siebtens geht es darum, „die Zukunft sichern“ [1, S. 295-303] zu helfen, soweit es geht. Das Leben ist grundsätzlich angefochten und unsicher. Der Mensch aber hat Sehnsucht nach äußerer und innerer Sicherheit. Äußere Sicherheit ist nur zum Teil herzustellen. Daher ist es notwendig, zu versuchen, eine gewisse innere Sicherheit zu erlangen. Hier sollte der Ort der religiösen Erziehung sein.

\section{Kindesentwicklung und Religion}

Der Mensch ist von Haus aus religiös. Er ist aufgrund seiner Geistesstruktur auf das Absolute ausgerichtet. Sonst könnte er - so hat es der Philosoph Hegel ausgedrückt - das Endliche nicht als endlich und das Relative nicht als relativ erfassen. Er kann dies nur, weil er immer schon im Raum des Absoluten steht. Die
Frage ist nur, wer oder was dieses Absolute ist. Und da gibt es nicht so viele Möglichkeiten. Das Absolute kann Eshaft sein wie ein Schicksal, es kann Duhaft sein wie ein persönlicher Gott oder aber unbestimmt bzw. apersonal, wie in asiatischen Religionen.

Eine gute Religiosität sollte dem Menschen einen inneren Halt verschaffen sowie eine gewisse Sicherheit, die äußerlich nicht zu erreichen ist. Eine religiöse Erziehung sollte dem jungen Menschen ein tieferes Seinsvertrauen (Gottvertrauen) vermitteln. Da der Mensch, wie gesagt, schon im Raum des Absoluten steht, sollte gerade Kindern eine religiöse Erziehung nicht vorenthalten werden. Kinder fragen nach den letzten Dingen, nach dem Tod, nach dem Himmel, nach Gott. Insofern hat das Kind ein „Recht“ auf eine religiöse Erziehung bzw. die Vermittlung religiöser Werte und Perspektiven. Kinder stellen Eltern religiöse Fragen und werden durch andere Kinder mit solchen Fragen konfrontiert. Sie kommen im Alltag mit der Religion anderer Kinder in Berührung. Ferner werden sie in der Musik, in der Literatur, in der Kunstgeschichte sowie im Alltag, wenn sie z. B. eine Kirche, eine Moschee, eine Synagoge, einen Tempel sehen, mit der Frage nach dem Religiösen konfrontiert. Man sollte sie deshalb nicht um die Möglichkeiten der Frage nach Gott „betrügen“, so nach dem Motto, sie sollen später selbst einmal entscheiden, ob sie sich dieser Frage zuwenden wollen. Die Frage ist existenziell da und drängt sich gewissermaßen auf. Auch angesichts des Todes und der Frage nach dem Sinn des Lebens ist sie präsent. Schließlich ist religiöse Bildung Teil einer guten Allgemeinbildung.

Freie Entscheidungen für oder gegen eine Religionszugehörigkeit kann man nur treffen, wenn man informiert ist. Man muss sich konkret auseinandersetzen mit den existenziellen Fragen. Es ist auch für die Eltern eine Chance, sich neu mit ihrer eigenen Tradition zu befassen. Begriffe wie Menschenwürde, Solidarität, Subsidiarität, Nächstenliebe, Feindesliebe sowie die strukturellen Errungenschaften wie Arbeitslosengeld, Krankenkassen, Pensionsversicherungen haben im Letzten einen christlichen Hintergrund mit der Maxime, auch dem 
Benachteiligten, Armen, Kranken, alten Menschen zu helfen. So hat die religiöse Dimension immer mit Spiritualität und dem Innenleben des Menschen sowie mit ethischer Umsetzung im Miteinander der Menschen zu tun. Kinder sollten zum respektvollen Umgang mit Andersdenkenden und Andersgläubigen erzogen werden.

Es wäre wünschenswert, den Kindern die ganze große Welt des Geistes, des Denkens und Fühlens zu eröffnen, die weit über das naturwissenschaftlich Erforschbare hinausragt. Es geht darum, Neugierde bei den Kindern zu wecken. Immanuel Kant sagte: „Zwei Dinge erfüllen das Gemüt mit immer neuer und zunehmender Bewunderung und Ehrfurcht, je öfter und anhaltender sich das Nachdenken damit beschäftigt: Der bestirnte Himmel über mir, und das moralische Gesetz in mir" [4].

Die Welt ist mehr als das, was man mit den Händen be-greifen und mit naturwissenschaftlicher Forschung erfassen kann. Es gilt, jungen Menschen diesen weiten Blick und ein gutes Grundvertrauen, Urvertrauen, Gottvertrauen zu vermitteln. Das Vertrauen beginnt mit den menschlichen Kontakten, sollte aber darüber hinausweisen. Das Neue Testament beschreibt diesen Überstieg in der Geschichte von Jesus Christus, der im Alter von 12 Jahren im Tempel zurückbleibt und die Eltern ihn suchen. Auf ihre Vorwürfe hin, dass er verschwunden sei, sagt er lapidar: „Wusstet ihr nicht, dass ich in dem sein muss, was meines Vaters ist" (Lk 2,49). Er wendet sich hier von seinen Eltern ab und seinem göttlichen Urgrund zu. Damit übersteigt er die rein innerweltliche Ebene. Zur Reifung von Kindern ist dieser Überstieg in der Pubertät von großer Bedeutung. Der junge Mensch soll Halt finden in einem anderen Grund, den die Tradition Seelengrund nennt. Das Christentum spricht hier vom göttlichen Grund im Menschen, der dem Einzelnen von innen her Halt geben kann. Von dort her findet der Mensch seinen eigentlichen Selbststand, seine Wahrheit, Berufung und Identität.

Auf das ganze Leben bezogen ist Religion der Ort, wo Menschen geöffnet werden können für die großen Fragen, die

Paediatr. Paedolog. 2020 · 55 (Suppl 2):S58-S61 https://doi.org/10.1007/s00608-020-00779-0

(c) Der/die Autor(en) 2020

\title{
M. Beck \\ Ethische Fragen zum Kindeswohl: Gelingen und Misslingen von Entwicklung
}

\begin{abstract}
Zusammenfassung
Kinder sind sehr vulnerabel. Für eine gute Entwicklung benötigen sie stabile Beziehungen und gute Umgebungsbedingungen. Sie haben ein Bedürfnis nach körperlicher Unversehrtheit und Sicherheit sowie nach Erfahrungen, die auf ihre individuellen Unterschiede zugeschnitten sind. Wichtig ist das Wissen um ihre Herkunft. Weiterhin wollen sie Erfahrungen machen, die ihrem Entwicklungsstand angemessen sind. Sie brauchen Grenzen und Strukturen, unterstützende Gemeinschaften und kulturelle Kontinuität. Diese sollen ihnen helfen, ihre ungesicherte Zukunft zu sichern. Allerdings
\end{abstract}

\section{Ethical Questions on Child Welfare: Success and Failure of Development}

\section{Abstract}

Children are very vulnerable. For good development they need stable relationships and secure family settings. They have a need for physical integrity and security as well as experiences that are tailored to their individual differences. It is important that they know about their origins. Furthermore, they want to gain experience appropriate for their stage of development. They need structures and boundaries, supportive communities, and cultural continuity. All this should help them to protect their unsecure future. Nevertheless, bleibt die Welt endlich und ungesichert. Eine stabile Sicherung erlangt man nur im eigenen Inneren. Hier ist der Ort des Religiösen. Eine gute religiöse Erziehung sollte gerade jungen Menschen einen festen Eigenstand vermitteln. Sie haben ein Recht darauf, ihre existenziellen Fragen nach dem Sinn des Lebens und nach den letzten Dingen des Seins beantwortet zu bekommen.

\section{Schlüsselwörter}

Philosophie · Theologie · Religion · Autorität Menschenwürde auf das Entdecken des Geheimnisvollen und Unerklärlichen in dieser Welt abzielen. Die Welt ist mehr als man hören und sehen kann. Die Welt ist nicht nur im empirisch Nachprüfbaren wahrzunehmen, sondern auch auf andere Weise. Es gibt die Erfahrung der bedingungslosen Annahme, die aufheilsame Weise mit Fragen nach dem Absoluten verbunden werden können. Wie überall gibt es auf der negativen Seite auch berechtigte Anfragen, wie Kinder vor verfehlter religiöser Erziehung geschützt werden können. the world remains finite and unsafe. Stable security can only be achieved within oneself. This is the place for religion. A good religious upbringing should give young people in particular inner stability. They have a right to get answers to existential questions regarding the sense and the meaning of life.

\section{Keywords}

Philosophy · Theology · Religion · Authority · Dignity

\section{Kindesentwicklung, Babyklap- pe und In-vitro-Fertilisation}

Wie schon in einem vorigen Beitrag beschrieben [5], geht es im Kontext der Babyklappe sowie in ganz anderem Kontext auch bei der In-vitro-Fertilisation darum, das Bestmögliche für die Entwicklung des Kindes zu tun. Bei der In-vitro-Fertilisation besteht die Gefahr, dass die Kinder z. B. ihre genetischen Eltern nicht kennen (Samenspende, Eizellspende). In Österreich haben sie mit 16 Jahren das Recht, den genetischen Vater oder die genetische Mutter kennenzulernen. Es kann auch sein, dass ein Kind mehrere Eltern hat. Dies ist zum Beispiel der Fall, wenn im Zuge einer gleichgeschlechtlichen Partnerschaft die 
Frau, die das Kind austrägt, zwar rechtlich die Mutter ist, das Kind aber aus einer Eizellspende der Lebenspartnerin und einer Samenspende zustande gekommen ist. Die Frau, die das Kind austrägt, ist auch rechtlich die Mutter, aber genetisch ist die Lebenspartnerin die Mutter und der Samenspender der Vater. Bei gleichgeschlechtlichen Partnerschaften ist die Frau, die das Kind austrägt, die Mutter, die Lebenspartnerin kann aber nicht der Vater sein. Also muss man die Gesetze ändern und von Eltern 1 und Eltern 2 sprechen.

Für die Identitätsfindung des Kindes ist die Kenntnis des genetischen Vaters und der genetischen Mutter von großer Bedeutung. Selbst wenn Studien auszusagen scheinen, dass ein Kind bei gleichgeschlechtlichen Eltern genauso gut aufwächst wie bei einem heterosexuellen Paar, scheint doch die Polarität des Männlichen und Weiblichen in der elterlichen Beziehung für die Entwicklung des Kindes förderlich zu sein. Ein Kind braucht bestimmte Grundbedingungen, um gesund aufwachsen zu können. Es hat ein Recht darauf, dass seine Bedürfnisse ernst genommen werden. Eine gute Sozialisierung von Kindern und Jugendlichen ist zentral für das Gelingen eines Lebens.

Ähnlich und doch ganz anders ist es bei einer anonymen Kindesabgabe (Babyklappe), bei der das Kind später gar keinen Zugang zu seiner Mutter oder seinem Vater hat. Wenn das Kind seine Eltern nicht kennt, sind die Bedingungen für eine gute Kindesentwicklung nicht optimal. Die sieben genannten Bedürfnisse müssten um jene des Wissens um die Herkunft des Kindes ergänzt werden. Dieses Wissen ist für eine gute Kindesentwicklung und die Identitätsfindung von großer Bedeutung (vgl. dazu [6]). Auch für die Mutter (den Vater) eines Kindes, das anonym abgegeben wurde, ist es auf lange Sicht schmerzlich, wenn sie nicht wissen, was aus dem Kind geworden ist. Auch für sie wäre es gut, die Möglichkeit zu haben, nach einer gewissen Zeit - mit Zustimmung des Kindes - Kontakt zu ihm aufzunehmen. Eine solch vollständige anonyme Kindesabgabe ohne die Möglichkeit einer späteren Kontaktaufnahme von beiden Seiten wird deshalb auch vom deutschen Ethikrat abgelehnt (vgl. dazu [7]).

\section{Korrespondenzadresse}

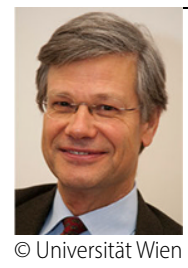

Univ. Prof. Dr. med. Dr.

theol. Mag. pharm.

Matthias Beck

Katholisch-theologische

Fakultät, Institut für

Systematische Theologie und

Ethik, Theologische Ethik,

Forschungsschwerpunkt

Medizinethik, Universität

Wien

Schenkenstraße 8-10,

1010 Wien, Österreich

Matthias.Beck@univie.ac.at

Funding. Open access funding provided by University of Vienna.

\section{Einhaltung ethischer Richtlinien}

Interessenkonflikt. M. Beck gibt an, dass kein Interessenkonflikt besteht.

Für diesen Beitrag wurden vom Autor keine Studien an Menschen oder Tieren durchgeführt. Für die aufgeführten Studien gelten die jeweils dort angegebenen ethischen Richtlinien.

Open Access. Dieser Artikel wird unter der Creative Commons Namensnennung 4.0 International Lizenz veröffentlicht, welche die Nutzung, Vervielfältigung, Bearbeitung, Verbreitung und Wiedergabe in jeglichem Medium und Format erlaubt, sofern Sie den/die ursprünglichen Autor(en) und die Quelle ordnungsgemäß nennen, einen Link zur Creative Commons Lizenz beifügen und angeben, ob Änderungen vorgenommen wurden.

Die in diesem Artikel enthaltenen Bilder und sonstiges Drittmaterial unterliegen ebenfalls der genannten Creative Commons Lizenz, sofern sich aus der Abbildungslegende nichts anderes ergibt. Sofern das betreffende Material nicht unter der genannten Creative Commons Lizenz steht und die betreffende Handlung nicht nach gesetzlichen Vorschriften erlaubt ist, ist für die oben aufgeführten Weiterverwendungen des Materials die Einwilligung des jeweiligen Rechteinhabers einzuholen.

Weitere Details zur Lizenz entnehmen Sie bitte der Lizenzinformation auf http://creativecommons.org/ licenses/by/4.0/deed.de.

\section{Literatur}

1. Brazelton TB, Greenspan SI (2002) Die sieben Grundbedürfnisse von Kindern: was jedes Kind braucht, um gesund aufzuwachsen, gut zu lernen und glücklich zu sein. Beltz, Weinheim

2. Spitzer M (2014) Digitale Demenz. Wie wir unsere Kinder um den Verstand bringen. Droemer, München
3. Beck M (2018) Was uns frei macht. Für eine Spiritualität der Entfaltung. Styria Verlag, Wien, Graz, Klagenfurt

4. Kant I (1995) Kritik der praktischen Vernunft. Reclam, Stuttgart (Kap. 34)

5. Beck M (2014) Identitätsfindung. Babyklappe und In-vitro-Fertilisation. Padiatr Padol 8:1-6

6. A. Lischka (Hrsg.), Eltern unbekannt. Auswirkungen auf die Identitätsfindung des Kindes. Pädiatrie \& Pädologie, Band 49, Supplement 2, Dezember 2014.

7. Deutscher Ethikrat (2009) Das Problem der anonymen Kindesabgabe. Deutscher Ethikrat, Berlin

Hinweis des Verlags. Der Verlag bleibt in Hinblick auf geografische Zuordnungen und Gebietsbezeichnungen in veröffentlichten Karten und Institutsadressen neutral. 